\title{
Florística e estrutura de fragmento florestal em área de transição na Amazônia Matogrossense no município de Sinop
}

\author{
Rosalia de Aguiar ARAUJO ${ }^{1}$, Reginaldo Brito da COSTA², Jeanine Maria FELFILI ${ }^{3}$, Ivani KUNTZ \\ Gonçalvez ${ }^{4}$, Roberto Antonio Ticle de Melo e SOUSA ${ }^{5}$, Alberto DORVAL ${ }^{6}$
}

\begin{abstract}
RESUMO
A fragmentação do ambiente é intensa na amazônia matogrossense e é esperado como consequência, além do desaparecimento da vegetaçáo original, que a flora dos fragmentos seja transicional, com elementos de floresta amazônica e de cerrado e que já apresentem elevada presença de famílias e espécies pioneiras. Este trabalho objetivou avaliar a composição florística e obter parâmetros fitossociológicos de componentes arbóreos presentes em um fragmento urbano no município de Sinop, Mato Grosso com vistas a confirmar sua classificação como vegetação de transição e detectar indícios de perturbação pela fragmentação. A vegetação foi amostrada pela instalação de 25 parcelas permanentes de $20 \times 20 \mathrm{~m}$, onde foram amostrados os indivíduos com CAP igual ou superior a $15 \mathrm{~cm}$ à altura de $1,30 \mathrm{~m}$ do solo. A densidade total da área foi de 1555 ind./ha, distribuídos em 37 famílias botânicas, 81 gêneros e 113 espécies. A família mais representativa foi Leguminosae com 14 espécies. As espécies que mais contribuíram em abundância e apresentaram maior frequência foram: Cecropia sciadophylla, Cecropia sp., Bellucia grossularioides e Vismia guianensis. O índice de Shannon $\left(\mathrm{H}^{\prime}\right)$ foi de 3,55, considerado alto para uma floresta de transição. A equabilidade de Pielou foi de 0,75 , sugerindo grande dominância de poucas espécies. As espécies que mais se destacaram em ordem decrescente de VI (\%) foram Cecropia sp., Bellucia grossularioides, Qualea ingens, Cecropia sciadophylla, Vismia guianensis, Miconia prasina, Trattinickia burserifolia, Unonopsis guatterioides e Schefflera vinosa. O remanescente apresenta uma flora mista amazônica e de cerrado, confirmando ser ecótono e a abundância de pioneiras sugere distúrbios. Esta floresta protege espécies madeireiras e frutíferas com grande potencial para uso múltiplo, podendo ser um espaço educativo com vistas a conservação e manejo sustentável.
\end{abstract}

PALAVRAS-CHAVE: Amazônia, cerrado, floresta estacional, floresta de transição, fitossociologia

\section{Floristics and structure of a forest fragment at a transitional zone at the Amazon in Mato Grosso State, Municipality of Sinop.}

\begin{abstract}
Fragmentation is intense in Amazon forest especially in Mato Grosso State. Loss of original vegetation is expected along with a transitional flora in such fragments, having elements of rainforest and Cerrado vegetation with abundant pioneer species. Goal of this work was to evaluate the floristic composition and phytosociology of the arboreal vegetation of an urban forest fragment in Sinop, Mato Grosso. It was intended to confirm its transitional characteristic and to detect possible perturbation due to fragmentation. The trial had $25(20 \times 20 \mathrm{~m})$ permanent plots, where all individuals over $15 \mathrm{~cm}$ gbh were assessed. A total of 36 families, 81 genera and 113 species were found in the area with 1555 ind.ha ${ }^{-1}$ plant density. The largest family was Leguminosae with 14 species. The most frequent species were: Cecropia sciadophylla, Cecropia sp., Bellucia grossularioides e Vismia guianensis. Shannon (H') diversity index reached 3.55 nats.ind ${ }^{-1}$, considered high for transitional forests. Pielou's evenness was 0.75 , suggesting high dominance of few species. In a decreasing ranking of VI (\%) the most frequent species were: Cecropia sp., Bellucia grossularioides, Qualea ingens, Cecropia sciadophylla, Vismia guianensis, Miconia prasina, Trattinickia burserifolia, Unonopsis guatterioides and Schefflera vinosa. The Amazon and Cerrado mixed floristic composition confirms the fragment as transitional and the abundance of pioneer species suggests disturbances caused by fragmentation.
\end{abstract}

KEYWORDS: Amazon, bioindicators, cerrado, seasonal forest, transitional forest.

\footnotetext{
1 Universidade Federal de Mato Grosso. E-mail: rosabiog@gmail.com

2 Universidade Federal de Mato Grosso. E-mail: reg.brito.costa@gmail.com

${ }^{3}$ Universidade de Brasília. E-mail: jeanine.felfili@pq.cnpq.br

${ }^{4}$ Universidade do Estado de Mato Grosso. E-mail: unemat@unemat.br

${ }^{5}$ Universidade Federal de Mato Grosso. E-mail: pgef|@ufmt.br

${ }^{6}$ Universidade Federal de Mato Grosso. E-mail: pgefl@ufmt.br
} 


\section{INTRODUÇÃO}

O avanço da atividade humana sobre a floresta determina a supressão da vegetaçáo o que Primack \& Rodrigues (2005), denominam de fragmentação do hábitat, processo em que uma grande e contínua área sofre reduçáo ou divisão. No Brasil Central (Felfili, 2003; Felfili et al., 2007) assim como nas transiçóes Cerrado Amazônia Marimon et al. $\left(2001^{\mathrm{a}, \mathrm{b}}\right)$ as Florestas Estacionais estâo sendo rapidamente convertidas em paisagens agrícolas, especialmente em pastagens.

$\mathrm{Na}$ região considerada como mata de transição (RADAMBRASIL, 1979; Alencar et al., 2004; Teixeira \& Rosendo, 2004; Villar et al., 2005; Carmo et al., 2006), entre Cerrado e Floresta Semidecídua, na Bacia do Teles Pires na pré-amazônia, o desmatamento e a consequente fragmentaçáo florestal foi ocasionado pela extraçáo da madeira e pela agricultura mecanizada. Apesar de raros estudos desenvolvidos, "este tipo vegetacional está desaparecendo rapidamente em funçáo de elevadas taxas de desmatamento provocadas pela atividade pecuária” (Kunz et al., 2008).

Os fragmentos florestais contêm uma parcela de representatividade da fauna e flora nativas, mas "geralmente, imersos em uma matriz fortemente antropizada" (Costa \& Scariot, 2003), que compromete o fluxo gênico e a própria sobrevivência do fragmento. Apresentam constantes mudanças em sua estrutura, fisionomia e composição florística, devido a alterações na matriz circundante. Segundo Primack \& Rodrigues, (2005) a quantificação das espécies existentes e como elas estão distribuídas são atividades básicas para delinear estratégias para conservar a diversidade biológica. Em um contexto temporal, a composição e a estrutura de um fragmento pode tender ao esperado para a biogeografia de ilhas (Primack \& Rodrigues, 2001), onde o fragmento representa uma ilha cercada por uma matriz inóspita alterada, suprimida e dividida pela construção da história e cultura humana.

Na pré-amazônia Mato Grossense, no Vale do Araguaia, Marimon et al. (2006) estudaram florestas semidecíduas e de interflúvio também fragmentadas. Nessa regiāo, Marimon et al. $\left(2001^{\mathrm{a}, \mathrm{b}}\right)$ constataram a monodominância de Brosimum rubescens, Moraceae. Mas, todos os estudos mostraram uma grande riqueza de espécies raras, muitas espécies comuns com as florestas ombrófilas assim como espécies comuns com as florestas estacionais do Cerrado. Outros autores, encontraram, em regiôes de transição como em Água Boa, MT (Felfili et al., 2000), em Nova Xavantina (Marimon et al. 2002), em Gaúcha do Norte (Ivanauskas et al. 2004) em Querência do Norte (Kunz et al., 2008) uma flora mista, com elementos da Amazônia e do Cerrado.

Essa flora mista, característica transicional, deve ser também encontrada neste fragmento. Indícios da fragmentação na flora, com abundância de espécies típicas de clareiras são também esperados. Espécies com características pioneiras, podem funcionar como bioindicadoras uma vez que a literatura mostra que, em condiçôes náo perturbadas, o percentual dessas espécies na estrutura comunitária é baixo, enquanto em áreas perturbadas elas podem ser dominantes (Felfili, 1997).

Estudos florísticos e fitossociológicos de fragmentos florestais, em áreas de transição entre Cerrado e Floresta Amazônica, podem subsidiar açôes que visem o manejo, a restauraçáo, a conservação e a implantaçáo de corredores de conexôes (Silva et al., 2004; Redling, 2007, Kunz, et al. 2008) tanto para a Amazônia quanto para o Cerrado. É esperado que em tal ambiente sejam encontradas tanto espécies de um quanto de outro bioma e também espécies restritas.

Os resultados obtidos poderáo contribuir para a recuperação da vegetação remanescente, tendo em vista a minimização do processo de supressão da vegetação e a preservação da sua biodiversidade, fomentando pesquisa e atividades ecoturísticas, considerando a viabilidade e sustentabilidade ambiental e sócio-econômica.

O presente trabalho objetivou avaliar a composição florística e obter parâmetros fitossociológicos de componentes arbóreos de um fragmento em área de transição no Município de Sinop, Mato Grosso.

\section{MATERIAL E MÉTODOS}

Local de estudo - A Reserva R-7, considerada Área de Preservação Permanente, está localizada no perímetro urbano do município de Sinop, Mato Grosso (1 $1^{\circ} 51^{\prime} 0,8^{\prime \prime}$ e e $\left.55^{\circ} 30^{\prime} 56^{\prime} \mathrm{W}\right)$. O fragmento com área de $45 \mathrm{ha}$, corresponde a uma floresta de transição entre Floresta Amazônica e Cerrado e acompanha o Córrego Marlene que contribui para a formação do Rio Teles Pires, considerado um dos mais importantes rios do Estado do Mato Grosso.

O clima da regiâo é tropical, quente e úmido (Am de Köeppen), com uma estação seca mais prolongada e uma estação úmida de quatro meses, entre dezembro a março. A precipitação pluviométrica média anual é de $2000 \mathrm{~mm}$, muito reduzida no período de maio a agosto. Apresenta uma temperatura média em torno de $24^{\circ} \mathrm{C}$.

A vegetação, segundo RADAMBRASIL (1979), é denominada de Floresta Semidecidual. Teixeira \& Rosendo (2004) denomina área de transição, que recobre a maior parte da bacia do Teles Pires, pontuada por trechos de savana, com grande potencial madeireiro.

A topografia é plana com solo do tipo latossolo vermelhoamarelo, caracterizado como solo mineral, variando de profundos a muito profundos, bem drenados, muito permeáveis e porosos. Esse tipo de solo apresenta deficiências minerais e baixa reserva de elementos nutritivos (Higuchi $e t$ al., 2004). 
Coleta de dados - Para a avaliação qualitativa e quantitativa dos elementos arbóreos da vegetação, foram alocadas vinte e cinco parcelas de $20 \mathrm{~m} \times 20 \mathrm{~m}\left(400 \mathrm{~m}^{2}\right)$, totalizando uma área amostral de 1,0 ha. As parcelas foram distribuídas de forma sistemática contemplando os diferentes estágios de sucessáo ecológica de modo que 15 parcelas foram delimitadas em uma área do fragmento onde se encontra mais preservada e 10 em área bem alterada, inclusive com histórico de queimadas sucessivas (Fig. 1).

No interior das parcelas foram numerados seqüencialmente todos os indivíduos vivos ou mortos em pé, com circunferência à altura do peito - CAP (1,30 $\mathrm{m}$ do solo) igual ou superior a $15 \mathrm{~cm}$, onde foram obtidas a variável CAP e a altura com uma vara de referência de 6 metros.

Para a identificação taxonômica, foi utilizado o sistema de classificação de Cronquist (1981), exceto para as famílias Caesalpiniaceae, Papilionaecae e Mimosaceae que foram tratadas como subfamílias da família Leguminosae. Foi efetuada coleta florística de espécies arbóreas e arbustivas no período de dezembro de 2006 a janeiro de 2008. A identificação do material botânico foi realizada por meio de comparaçóes com material depositado no herbário da Universidade Federal de Mato Grosso (UFMT) em Cuiabá, por especialistas e à literatura especializada. A confirmaçáo e atualização das denominações e autorias das espécies amostradas foram realizadas através do banco de dados do Missouri Botanical Garden (http://mobot.mobot. Org).
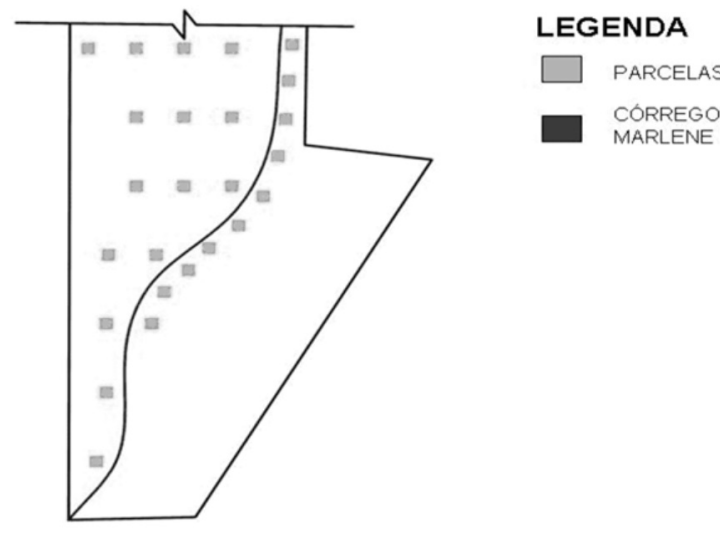

Figura 1- Croqui da distribuição das parcelas na Reserva R7 em Sinop, Mato Grosso

Foram estimados os seguintes parâmetros fitossociológicos (Mueller-Dombois \& Ellenberg, 1974, Kent \& Coker, 1992): densidade absoluta e relativa, frequência absoluta e relativa, dominância absoluta e relativa e valor de importância.

A diversidade alfa foi estimada pelo índice de Shannon $\left(\mathrm{H}^{\prime}\right)$ e a equabilidade foi calculada pelo índice de Pileou (J') (Brower \& Zar, 1984) e foi elaborada a curva espécie-área, com o uso do software Mata Nativa 2.06 (CIENTEC, 2006).

\section{RESULTADOS E DISCUSSÃO}

A amostra do componente arbóreo da reserva R7 de Sinop, Mato Grosso, apresentou 1.555 indivíduos dos quais 60 mortos e dois não identificados, pertencentes a 81 gêneros, 37 famílias botânicas e 113 espécies (Tab. 1).

As famílias que mais contribuíram para a riqueza florística foram: Leguminosae com 14 espécies (duas Caesalpinoideae, nove Mimosoideae e três Papilionoidaea), Annonaceae com 10, Euphorbiaceae com nove, Lauraceae com oito, Burseraceae com seis, Cecropiaceae, Melastomataceae, Sapindaceae com cinco, Rubiaceae e Clusiaceae com quatro cada. Essas nove famílias juntas respondem por $69,91 \%$ das espécies amostradas. As demais 27 famílias (incluindo os mortos e não identificados) contribuíram com 30,09\% do total.

A família Leguminosae foi a que apresentou maior diversidade de espécies, assim como nos estudos realizados em áreas de transição (Ivanauskas et al., 2002; R. A.Medeiros, dados não publicados), no pantanal (Marimon et al., 2006), em florestas estacionais (Felfili et al., 2000; Silva et al., 2004; Pereira-Silva et al., 2004; Damasceno Junior et al., 2005; Redling et al., 2002), e no cerrado (Andrade et al., 2002; Haidar et al., 2005 \& M. M. Barbosa, dados não publicados). A expressividade dessa família é marcante em estudos que consideram a baixa condição de fertilidade natural dos solos, o que, segundo Silva et al., (2004), se deve provavelmente à capacidade de fixação de nitrogênio apresentada por algumas espécies desta família.

A abundância de Lauraceae indica a proximidade da flora com a região amazônica, uma vez que essa família em geral é menos comum nas formaçôes de cerrado (Mendonça et al., 2008). No entanto, a única espécie de Caryocaraceae é típica do cerrado. Desse modo, a composição florística dessa área é compatível com a sugestấo de Odum (1988), onde as comunidades de ecótonos contêm muitos dos organismos de cada comunidade superposta e de organismos característicos de áreas de tensão e que frequentemente a riqueza e a abundância de algumas espécies são maiores no ecótone do que nas áreas adjacentes.

A elevada abundância de Cecropiaceae no presente estudo em comparaçấo com menores números encontrados em locais pouco perturbados no Cerrado e Floresta Amazônica (Silva et al., 2008) sugere o efeito de perturbaçóes no fragmento. Essa família foi registrada expressivamente em outras áreas de tensão ecológica ou área de transição, como nos municípios de Cláudia (Medeiros, 2004), com 16,21\% dos indivíduos e em Gaúcha do Norte, com uma espécie e três indivíduos (Ivanauska et al., 2004), mostrando que ela pode dominar ou não em ecótonos, reforçando assim a sugestão de que esta é uma bioindicadora de distúrbios em florestas (Felfili, 1997). 
Tabela 1 - Espécies e respectivas abundâncias registradas na Reserva R7 em Sinop, Mato Grosso, dispostas em ordem alfabética de famílias botânicas e acompanhadas de seus respectivos nomes populares.

\begin{tabular}{|c|c|c|}
\hline Famílias/Espécies & Nome Popular & $\mathrm{N}^{0}$ \\
\hline ANACARDIACEAE & & 63 \\
\hline Astronium sp. & amescla rosa 2 & 3 \\
\hline Tapirira cf. guianensis Aubl. & amescla & 37 \\
\hline Thyrsodium spruceanum Benth. & ----------- & 23 \\
\hline ANNONACEAE & & 95 \\
\hline Bocageopsis multiflora (Mart.) R.E.Fr. & envira surucucu & 1 \\
\hline Cardiopetalum calophyllum Schtdl. & peroba 1 & 3 \\
\hline Guatteria cauliflora Mart. & Pindaíba & 10 \\
\hline Guatteria nigrescens Mart. & Pindaíba preta 2 & 2 \\
\hline Guatteria sp. & Envira & 2 \\
\hline Duguetia lanceolata A. St.-Hil & Pindaíva & 3 \\
\hline Duguetia sp & ------------ & 2 \\
\hline Unonopsis guatterioides (A.DC) R.E.Fr. & Correera & 58 \\
\hline Xylopia benthamii R.E.Fr. & Pindaíba preta & 13 \\
\hline Xylopia emarginata Mart & envira folha fina & 1 \\
\hline APOCYNACEAE & & 15 \\
\hline Aspidosperma sp. & peroba & 9 \\
\hline Himatanthus sucuuba (Spruce ex Müll. Arg.) Woodson & Leiteiro & 6 \\
\hline ARALIACEAE & & 35 \\
\hline Schefflera morototoni (Aubl) Maguire, Steyerm.\& Frodin & ------------- & 5 \\
\hline Schefflera vinosa (charm. \& Schitdl.) Frondin \& Fiaschi & Mandiocão & 30 \\
\hline BIGNONIACEAE & & 31 \\
\hline Jacaranda copaia (Aubl.)D.Don & Marupá & 31 \\
\hline BOMBACACEAE & & 3 \\
\hline Bombacopsis glabra (Pasq.)Robyns & castanha do Maranhão & 1 \\
\hline Eriotheca gracilipes (K.Schum.)A.Robyns & paineira branca & 2 \\
\hline BORAGINACEAE & & 17 \\
\hline Cordia alliodora (Ruiz \& Pav.) Cham. & -------------- & 2 \\
\hline Cordia bicolor A.DC. & pau rolha & 14 \\
\hline Cordia nodosa Lam. & baba de boi & 1 \\
\hline BURSERACEAE & & 88 \\
\hline Protium aracouchini (Aubl)Marchand & amescla 4 & 7 \\
\hline Protium heptaphyllum (Aubl)Marchand & amescla 3 & 4 \\
\hline Protium pilosum (Cuatrec.) D.C.Daly & amesclinha & 2 \\
\hline Protium sp. & ----------------- & 2 \\
\hline Tetragastris sp. & Pindaíba 1 & 3 \\
\hline Trattinnickia burserifolia Mart. & amescla rosa & 70 \\
\hline CARYOCARACEAE & & 3 \\
\hline Caryocar brasiliense Cambess & Piqui da mata & 3 \\
\hline CECROPIACEAE & & 416 \\
\hline Cecropia latiloba Miq. & embaúba preta & 42 \\
\hline Cecropia sciadophylla Mart. & embaúba branca & 89 \\
\hline Cecropia sp. & Embaúba & 281 \\
\hline Pouroma cecropiifolia Mart. & Imbaúba & 3 \\
\hline Pourouma velutina Mart.ex Miq. & Figueira & 1 \\
\hline CELASTRACEAE & & 7 \\
\hline Goupia glabra Aubl. & Cupiúba & 7 \\
\hline CLUSIACEAE & & 98 \\
\hline Calophyllum brasiliense Cambess. & --------------- & 1 \\
\hline Rheedia sp. & Bacopari & 2 \\
\hline
\end{tabular}


Tabela 1 - Continuação

\begin{tabular}{|c|c|c|}
\hline Famílias/Espécies & Nome Popular & $\mathrm{N}^{0}$ \\
\hline Vismia brasiliensis Choisy & Guatambú & 4 \\
\hline Vismia guianensis (Aublet.) Choisy & café bravo & 91 \\
\hline CRYSOBALANACEAE & & 1 \\
\hline Couepia sp. & Murta & 1 \\
\hline ELAEOCARPACEAE & & 14 \\
\hline Sloaneae guianensis (Aubl.)Benth & Pateiro & 14 \\
\hline EUPHORBIACEAE & & 57 \\
\hline Alchornea discolor Poepp. & amescla do banhado 1 & 9 \\
\hline Alchornea triplinervia (Spreng)Müll.Arg. & Gaivotinha & 4 \\
\hline Aparisthmium cordatum Baill. & Marmeleiro & 1 \\
\hline Croton sp. & Capixingui & 12 \\
\hline Hevea brasiliensis (Willd.ex A.Juss.)Müll.Arg. & Seringueira & 5 \\
\hline Hyeronima alchorneoides Allem. & Margonçalo & 1 \\
\hline Mabea fistulifera Mart. & leiteiro branco & 21 \\
\hline Pera glabrata (Schott.) Poepp ex Baill. & Caferana & 1 \\
\hline Sapium glandulosum (L.)Morong & leiteiro da casca grossa & 3 \\
\hline HIPPOCRATEACEAE & & 1 \\
\hline Cheiloclinium cognatum (Miers)AC.Sm. & --------------- & 1 \\
\hline LACISTEMATACEAE & & 1 \\
\hline Lacistema pubescens Mart. & Apuizinho & 1 \\
\hline LAURACEAE & & 73 \\
\hline Aniba ferrea kubizki & canelão rosa & 2 \\
\hline Mezilaurus itauba (Meisnn.)Taub.ex Mez & Itaúba & 1 \\
\hline Nectandra sp. & Louro & 3 \\
\hline Ocotea glomerata (Nees.)Mez. & Amescla & 6 \\
\hline Ocotea guianensis Aubl & canela serrafaz & 13 \\
\hline Ocotea sp. & canela amarela & 16 \\
\hline Ocotea Suaveolens (Meisn.)Hassl. & canela rosa & 30 \\
\hline Persea cordata Meisn. & abacateiro bravo & 2 \\
\hline LEGUMINOSAE - CAESALPINOIDEAE & & 8 \\
\hline Tachigali sp. & cravão branco & 3 \\
\hline Tachigali venusta Dwyer & 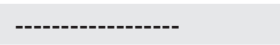 & 5 \\
\hline LEGUMINOSAE - MIMOSOIDEAE & & 96 \\
\hline Abarema jupunba Willd. & 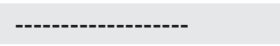 & 1 \\
\hline Inga dysantha Benth & ingá peludo & 3 \\
\hline Inga fagifolium Spruce ex Bth & ingá 4 & 15 \\
\hline Inga lateriflora Miq. & ingá 3 & 23 \\
\hline Inga marginata Willd. & Ingazinho & 25 \\
\hline Inga sp. & ingá rosa & 16 \\
\hline Inga vera ssp.affinis (DC.)T.D.Pennington & Ingá & 1 \\
\hline Parkia sp. & Muse & 10 \\
\hline Pithecellobium sp. & ingá de macaco & 2 \\
\hline LEGUMINOSAE - PAPILIONOIDEAE & & 6 \\
\hline Bowdichia virgilioides Kunth & sucupira preta & 1 \\
\hline Dalbergia sp. & Jacarandá & 1 \\
\hline Dipteryx odorata (Aubl.)Willd. & Champanhe & 4 \\
\hline MALPIGHIACEAE & & 13 \\
\hline Byrsonima sp. & Murici 4 & 1 \\
\hline Byrsonima spicata (Cav.)DC & murici & 12 \\
\hline MELASTOMATACEAE & & 258 \\
\hline
\end{tabular}


ACTA

AMAZONICA

Florística e estrutura de fragmento florestal em área de transição na Amazônia

Matogrossense no município de Sinop

Tabela 1 - Continuação

\begin{tabular}{|c|c|c|}
\hline Famílias/Espécies & Nome Popular & $\mathrm{N}^{0}$ \\
\hline Bellucia grossularioides (L.)Triana & jambo & 140 \\
\hline Miconia splendens (Sw.)Griseb. & Espeteiro & 31 \\
\hline Miconia prasina (Sw.)DC. & murici 1 & 82 \\
\hline Miconia sp. & erva de bicho & 3 \\
\hline Mouriri sp. & Muiraúba & 2 \\
\hline MELIACEAE & & 8 \\
\hline Trichilia pallida Sw. & Gitorana & 8 \\
\hline MENISPERMACEAE & & 7 \\
\hline Abuta concolor Poepp.\& Endl. & grão de galo & 6 \\
\hline Abuta grandifolia (Mart.)Sandw. & pau de ferro & 1 \\
\hline MONIMIACEAE & & 3 \\
\hline Siparuna guianensis Aubl. & Negramina & 3 \\
\hline MORACEAE & & 5 \\
\hline Pseudolmedia laevigata Trec. & larga galha & 2 \\
\hline Sorocea guilleminiana Gaudich. & bainha-de-espada & 3 \\
\hline MYRISTICACEAE & & 7 \\
\hline Virola calophylla (Spruce)Warb. & --------------- & 7 \\
\hline MYRTACEAE & & 7 \\
\hline Calyptranthes paniculata Ruiz \& Pav. & pitanga & 1 \\
\hline Caliptranthes sp. & Goiabinha & 4 \\
\hline Eugênia sp. & Pitanga & 2 \\
\hline NYCTAGINACEAE & & 8 \\
\hline Guapira opposita (Vell.)Reitz. & farinha seca do brejo & 4 \\
\hline Neea oppositifolia Ruiz \& Pav. & farinha seca & 4 \\
\hline POLYGONACEAE & & 2 \\
\hline Triplaris sp. & Novateiro & 2 \\
\hline QUIINACEAE & & 1 \\
\hline Quiina pteridophylla (Radlk.)Pires & ------------- & 1 \\
\hline RUBIACEAE & & 8 \\
\hline Duroia saccifera (Mart.ex Roem.\& Schult.) & piruí do mato & 1 \\
\hline Palicourea guianenis Aubl. & erva de bicho bravo & 4 \\
\hline Palicourea sp. & ---------------- & 2 \\
\hline Psychotria capitata Ruiz \& Pav. & espeteiro branco & 1 \\
\hline RUTACEAE & & 2 \\
\hline Fagara rhoifolia (Lam.) Engl. & Mama de porca & 2 \\
\hline SAPINDACEAE & & 12 \\
\hline Alophyllus edulis Radlk. & -------------- & 2 \\
\hline Cupania castaneifolia Mart. & vara & 1 \\
\hline Cupania sp. & ---------------- & 2 \\
\hline Talisia sp. & Pitomba & 6 \\
\hline Toulicia sp. & Pitombinha & 1 \\
\hline SAPOTACEAE & & 5 \\
\hline Ecclinusa ramiflora Mart. & --------------- & 3 \\
\hline Ecclinusa sp. & Balata & 1 \\
\hline Micropholis sp. & --------------- & 1 \\
\hline TILIACEAE & & 1 \\
\hline Apeiba echinata Gaertn. & pente de macaco & 1 \\
\hline
\end{tabular}


Tabela 1 - Continuação

\begin{tabular}{llc}
\hline Famílias/Espécies & Nome Popular & $N^{0}$ \\
\hline ULMACEAE & & 1 \\
Trema micrantha (L.)Blume & Mirindiba & 1 \\
VOCHYSIACEAE & Marfim & 17 \\
Erisma sp. & Cambará & 6 \\
Qualea ingens Warm. & & 11 \\
TOTAL DE INDIVÍDUOS VIVOS & & 1.493 \\
TOTAL DE MORTOS & & 60 \\
TOTAL DE NÃO IDENTIFICADOS (NI) & 2 \\
TOTAL GERAL & & 1.555 \\
\hline
\end{tabular}

As famílias Cecropiaceae e Melastomataceae compreenderam $43,07 \%$ do total de indivíduos, apesar de apresentarem apenas cinco espécies cada. O gênero Cecropia representa 26,58\% em abundância, inclusive com a espécie que mais apresentou indivíduos, Cecropia sp. (281), contribuindo com 17,96 \%, demonstrando a ampla ocupação territorial dessa espécie no fragmento (Fig. 2). As outras nove espécies que apresentaram o maior número de exemplares foram: Bellucia grossularioides (140 ou 8,94\%), Vismia Guianensis (91 ou 5,81\%), Cecropia sciadophylla (89 ou 5,69\%), Miconia prasina (82 ou 5,24\%), Trattinickia burserifolia (70 ou 4,47\%), Unonopsis guatterioides (58 ou 3,70\%), Croton sp. (57 ou 3,64\%), Cecropia latiloba

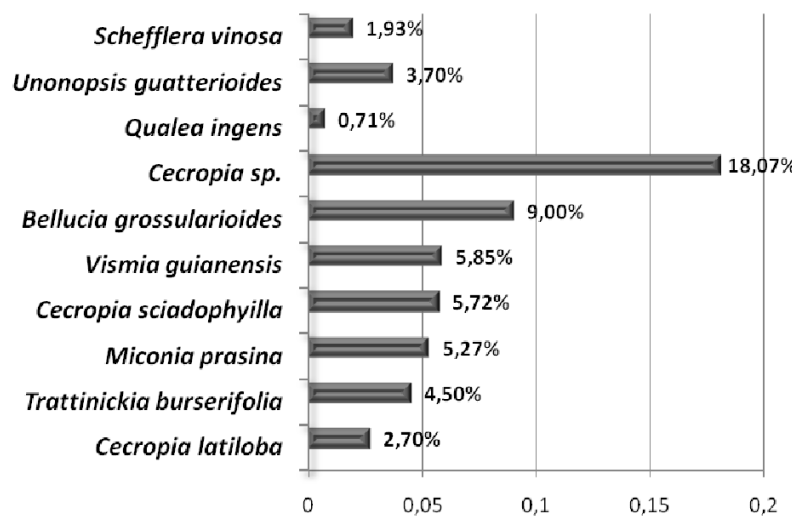

Figura 2 - Percentual do número de indivíduos das principais espécies amostradas na Reserva R7 em Sinop, Mato Grosso, número total=1555 indivíduos.

(42 ou 2,68\%), incluindo os indivíduos mortos com 60 exemplares ou $3,83 \%$ do total.

Em alguns levantamentos florísticos, as famílias que apresentam maior abundância também apresentam maior riqueza (Kunz et al., 2008; Oliveira et al., 2005), porém na Reserva R7 as duas famílias (Cecropiaceae e Melastomataceae) que apresentaram alta densidade, responsáveis por quase $50 \%$ do total de indivíduos, apresentaram pequeno número de gêneros e espécies (Fig. 3). Ivanauska et al., (2004), para uma área semelhante em Gaúcha do Norte no Mato Grosso, afirmam que algumas espécies têm maior facilidade em dominar o ambiente, principalmente aquelas com alto potencial de competiçáo que inibem o estabelecimento de várias outras. Neste caso, Cecropiaceae, especialmente o gênero Cecropia é uma espécie pioneira em florestas (Felfili

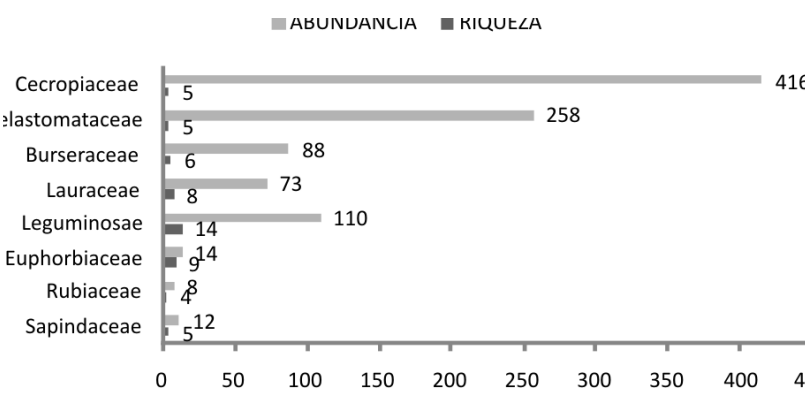

Figura 3 - Número de indivíduos (Abundância) e espécies (Riqueza) por família, amostrados na Reserva R7, em Sinop, Mato Grosso.

1997), sendo necessário ainda investigar as características funcionais das espécies de Melastomataceae.

Padrōes de abundância são bastante variáveis para espécies e famílias de um modo geral. Para Amaral et al., (2000), a abundância de indivíduos nem sempre é proporcional ao número de espécies, ou seja, poucas espécies podem ser representadas por grandes populaçôes ou uma única espécie pode ser muito abundante na comunidade. Essa observação é extensiva também, às famílias, que podem apresentar alta riqueza, mas serem pouco abundantes.

$\mathrm{O}$ gênero Inga apresentou o maior número de espécies (seis), seguido por Ocotea com cinco e Protium com quatro, Guatteria, Cordia, Cecropia e Miconia com três espécies cada.

As leguminosas, de acordo com Nunes et al., (2007), constituem uma das famílias de plantas mais importantes da Amazônia. O gênero Inga, é importante na recomposição e recuperação de áreas degradadas e florestas ciliares (Mata \& Felix, 2007; Stein et al., 2007). A maioria das espécies desse gênero é encontrada em formaçóes de diferentes domínios vegetacionais sendo consideradas generalistas (Mata \& Felix, 2007). 
As florestas do Bioma Amazônico são representadas por poucas espécies abundantes e muitas espécies raras (Muniz et al., 1994 (b); Ivanauska et al., 2004), contribuindo significativamente para o cômputo da diversidade florística de uma comunidade. Conforme Ivanauskas \& Rodrigues (2000), espécies com abundância intermediárias são aquelas que possuem de dois a nove indivíduos por ha e abundantes as que apresentam mais de 10 indivíduos por ha, as demais podem ser consideradas raras localmente.

Na Reserva R7 de Sinop, Mato Grosso, objeto de estudo deste trabalho, das 37 famílias botânicas identificadas, 58,32\% podem ser consideradas de baixa densidade $(71,43 \%$ dessas famílias foram consideradas raras). As raras compreendem $26 \%$, as intermediárias $46 \%$ e abundantes $28 \%$ da riqueza arbórea desse fragmento.

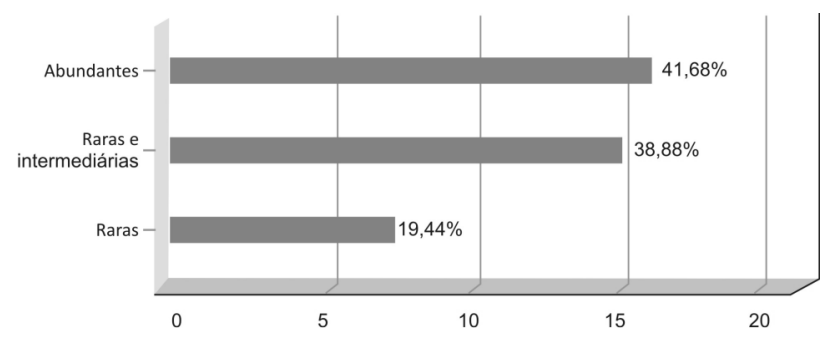

Figura 4 - Porcentagem de famílias com espécies raras localmente, intermediárias e abundantes encontradas na Reserva R7, em Sinop, Mato Grosso.

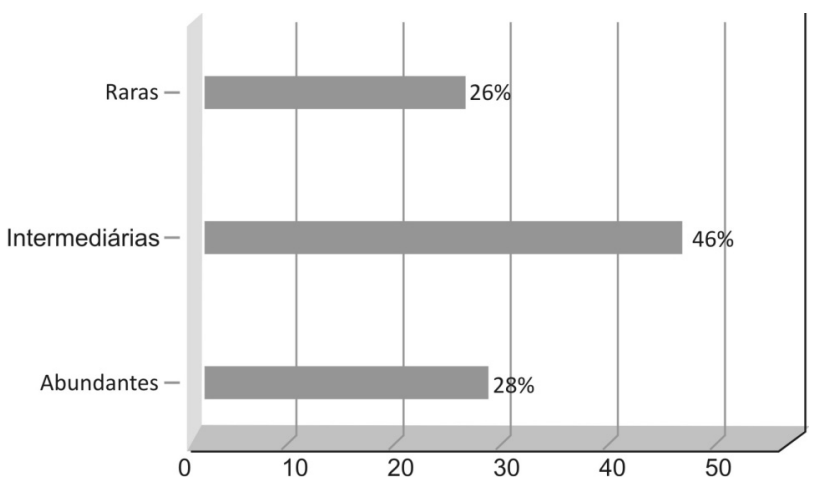

Figura 5 - Comparação entre as espécies raras, intermediárias e abundantes da reserva $\mathrm{R} 7$ em Sinop, Mato Grosso.

A porcentagem de espécies consideradas raras com apenas um exemplar por hectare encontradas neste trabalho são semelhantes àqueles obtidos por Silva et al. (1992), Almeida et al. (1993), Oliveira \& Amaral (2004) para a região amazônica (Fig. 4 e 5).

A ocorrência de um grande número de espécies com poucos indivíduos é registrada na maioria dos trabalhos em florestas tropicais preservadas, como conseqüência da alta diversidade biológica (Cardoso-Leite et al., 2002).
O índice de diversidade de Shannon (H') encontrado na área estudada foi de 3,55 e a equabilidade de 0,75. Segundo Meira Neto e Martins (2000), o índice de diversidade varia entre 3,2 e 4,2 e a equabilidade entre 0,73 e 0,88 para as florestas semideciduais em Minas Gerais. Em floresta de transição Ivanauska et al. (2004), Kunz et al (2008) e Ferreira Júnior et al. (2008) encontraram valores de 3,07 a 3,38 e de 0,5 a 0,85 , respectivamente.

A diversidade acentuada pode ser justificada pela proximidade com a Floresta Amazônica, pois segundo Kunz et al. (2008) e Felfili \& Felfili (2001) o índice de diversidade para cerrados que se encontram nessas áreas, é mais acentuada. Por outro lado, comunidades com alta equidade possuem baixa dominância entre as espécies (Brower \& Zar, 1984; Magurran, 1988; Pinto-Coelho, 2002; Cullen Jr. et al., 2004). $\mathrm{Na}$ Reserva $\mathrm{R} 7$ a equidade alta evidencia a alta heterogeneidade florística.

As espécies amostradas, com suas respectivas estimativas dos parâmetros fitossociológicos da estrutura horizontal, em ordem decrescente de valor de importância (VI), estấo representados na Tab. 2.

As vinte espécies mais importantes da comunidade (Fig. 6), em valor de importância (VI(\%)), são: Cecropia sp., Bellucia grossularioides, Qualea ingens, Cecropia sciadophylla, Vismia guianensis, Miconia prasina, Trattinickia burserifolia, Unonopsis guatterioides, Schefflera vinosa, Cecropia latiloba, Tapirira cf. guianensis, Erisma sp., Ocotea suaveolens, Inga lateriflora, Miconia splendens, Inga marginata, Ocotea sp., Jacaranda copaia e Thyrsodium spruceanum e morta. Essas espécies mais as árvores mortas são responsáveis por $65,56 \%$ do referido valor de importância. O valor de VI de Cecropia sp. é o dobro de Bellucia grossularioides que está em segundo lugar, principalmente devido densidade encontrada. Já Qualea ingens se destaca em terceiro lugar por apresentar uma elevada dominância, não sendo táo preponderante em termos de densidade na comunidade.

Exceto para Qualea ingens, as dominâncias (Figura 6) pouco contribuíram na classificação do VI das espécies amostradas, pois as espécies de maior VI apresentaram elevada densidade e/ou freqüência, indicando que as mesmas se encontram presentes em toda a área de estudo e que a caracterização da área ocorre por um pequeno agrupamento de espécies.

As espécies com os maiores VI são pioneiras ou secundárias iniciais de acordo com Gandolfi et al., (1995), evidenciando o efeito da fragmentação e da elevada alteração antrópica, decorrentes de queimadas, impactos na vegetação e no solo causados por presença de diversas trilhas traçadas aleatoriamente ou inadequadamente e despejo de resíduos sólidos urbanos por meio de tubulaçáo e valetas de drenagem de águas pluviais do Município que desemboca dentro do córrego que percorre toda a área da Reserva R7. 


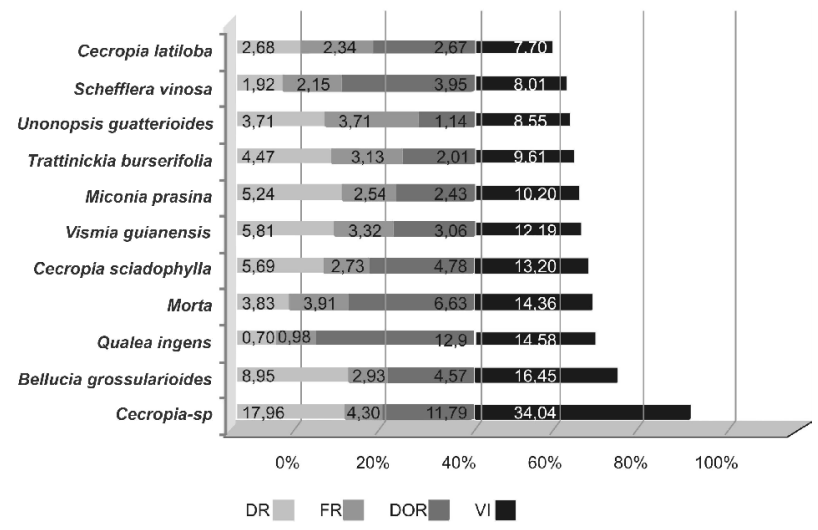

Figura 6 - Parâmetros fitossociológicos ( $F R=$ freqüência relativa; $D R=$ densidade relativa; $\mathrm{DoR}=$ dominância relativa) nas dez espécies de maior VI amostradas na Reserva R7 em Sinop, Mato Grosso.
A Fig. 7 representa o acúmulo do número de espécies com o número de parcelas ou unidades amostrais, mostrando que a tendência de estabilizaçáo da curva ocorreu a partir da $22^{\circ}$ parcela, atingindo a assíntota, confirmando que a amostra foi abrangente quanto à composição florística da área.

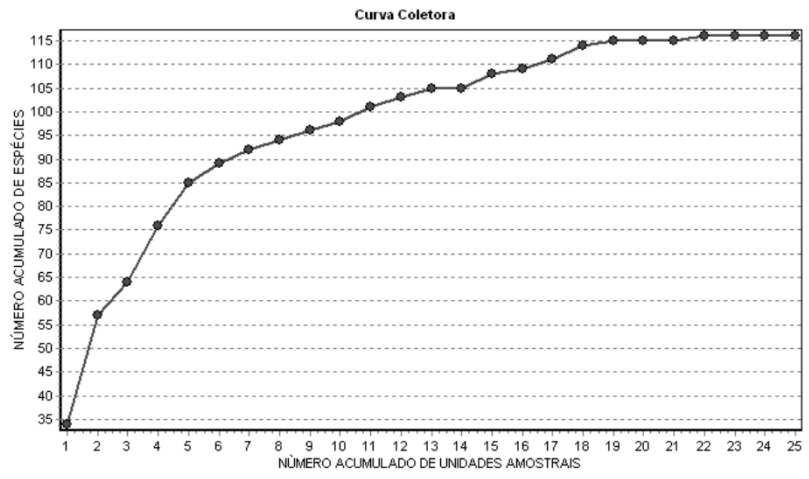

Figura 7 - Curva espécie-área da amostragem utilizada para caracterizar a floresta de transição na reserva R7, em Sinop, Mato Grosso.

Tabela 2 - Fitossociologia do estrato arbóreo da floresta de transição na Reserva R7 em Sinop, Mato Grosso, em ordem crescente do Índice de Valor de Importância. $\left(\mathrm{DA}=\right.$ densidade absoluta $\left(\mathrm{n} \cdot \mathrm{ha}^{-1}\right) ; \mathrm{DR}=$ densidade relativa $(\%) ; \mathrm{FA}=$ freqüência absoluta $(\%) ; \mathrm{FR}=$ frequiência relativa $(\%) ; D O A .=$ dominância absoluta ( $m^{2}$.ha-1); DOR= dominância relativa (\%); VI= Valor de Importância).

\begin{tabular}{|c|c|c|c|c|c|c|c|}
\hline Nome Científico & $\mathrm{DA}$ & $\mathrm{DR}$ & FA & FR & $\mathrm{DOA}$ & DOR & $\mathrm{VI}$ \\
\hline Cecropia sp. (Cecropiaceae) & 281,00 & 18,07 & 88,00 & 4,66 & 1,90 & 11,79 & 34,04 \\
\hline Bellucia grossularioides (Melastomataceae) & 140,00 & 8,95 & 60,00 & 2,93 & 0,73 & 4,57 & 16,45 \\
\hline Qualea ingens (Vochysiaceae) & 11,00 & 0,71 & 20,00 & 1,06 & 2,07 & 12,90 & 14,58 \\
\hline Morta & 60,00 & 3,86 & 80,00 & 4,23 & 1,06 & 6,63 & 14,36 \\
\hline Cecropia sciadophylla (Cecropiaceae) & 89,00 & 5,72 & 56,00 & 2,96 & 0,77 & 4,78 & 13,20 \\
\hline Vismia guianensis (Clusiaceae) & 91,00 & 5,85 & 68,00 & 3,60 & 0,49 & 3,06 & 12,19 \\
\hline Miconia prasina (Melastomataceae) & 82,00 & 5,27 & 52,00 & 2,75 & 0,39 & 2,43 & 10,20 \\
\hline Trattinnickia burserifolia (Burseraceae) & 70,00 & 4,50 & 64,00 & 3,39 & 0,32 & 2,01 & 9,61 \\
\hline Unonopsis guatterioides (Annonaceae) & 58,00 & 3,73 & 76,00 & 4,02 & 0,18 & 1,14 & 8,55 \\
\hline Schefflera vinosa (Apocynaceae) & 30,00 & 1,93 & 44,00 & 2,33 & 0,63 & 3,95 & 8,01 \\
\hline Cecropia latiloba (Cecropiaceae) & 42,00 & 2,68 & 48,00 & 2,54 & 0,43 & 2,67 & 7,70 \\
\hline Tapirira guianensis (Anacardiaceae) & 37,00 & 2,38 & 40,00 & 2,12 & 0,43 & 2,71 & 7,02 \\
\hline Erisma sp. (Vochysiaceae) & 6,00 & 0,38 & 12,00 & 0,63 & 0,84 & 5,25 & 6,22 \\
\hline Ocotea Suaveolens (Lauraceae) & 30,00 & 1,93 & 44,00 & 2,33 & 0,29 & 1,80 & 5,86 \\
\hline Inga lateriflora (Mimosoideae) & 23,00 & 1,48 & 60,00 & 3,17 & 0,18 & 1,13 & 5,53 \\
\hline Miconia splendens (Melastomataceae) & 31,00 & 1,99 & 44,00 & 2,33 & 0,14 & 0,92 & 5,04 \\
\hline Inga marginata (Mimosoideae) & 25,00 & 1,54 & 48,00 & 2,54 & 0,11 & 0,70 & 4,64 \\
\hline Ocotea sp. (Lauraceae) & 16,00 & 1,03 & 44,00 & 2,33 & 0,23 & 1,44 & 4,60 \\
\hline Jacaranda copaia (Bignoniaceae) & 31,00 & 1,99 & 24,00 & 1,27 & 0,22 & 1,39 & 4,53 \\
\hline Thyrsodium spruceanum (Anacardiaceae) & 23,00 & 1,48 & 44,00 & 2,33 & 0,10 & 0,62 & 4,24 \\
\hline Aspidosperma sp. (Apocynaceae) & 9,00 & 0,58 & 20,00 & 1,06 & 0,36 & 2,28 & 3,92 \\
\hline Pithecellobium sp. (Mimosoideae) & 2,00 & 0,13 & 8,00 & 0,42 & 0,52 & 3,25 & 3,77 \\
\hline Mabea fistulifera (Euphorbiaceae) & 21,00 & 1,35 & 24,00 & 1,27 & 0,15 & 0,93 & 3,44 \\
\hline Inga sp. (Mimosoideae) & 16,00 & 1,03 & 36,00 & 1,90 & 0,10 & 0,62 & 3,40 \\
\hline Inga fagifolium (Mimosoideae) & 15,00 & 0,96 & 36,00 & 1,90 & 0,08 & 0,54 & 3,25 \\
\hline Cordia bicolor (boraginaceae) & 14,00 & 0,90 & 32,00 & 1,69 & 0,11 & 0,73 & 3,18 \\
\hline Sloaneae guianensis (Elaeocarpaceae) & 14,00 & 0,90 & 32,00 & 1,69 & 0,05 & 0,36 & 2,81 \\
\hline Ocotea guianensis (Lauraceae) & 13,00 & 0,83 & 28,00 & 1,48 & 0,05 & 0,33 & 2,52 \\
\hline
\end{tabular}


Tabela 2 - Continuação

\begin{tabular}{|c|c|c|c|c|c|c|c|}
\hline Nome Científico & $\mathrm{DA}$ & $\mathrm{DR}$ & FA & $\mathrm{FR}$ & $\mathrm{DOA}$ & $\mathrm{DOR}$ & $\mathrm{VI}$ \\
\hline Alchornea discolor (Euphorbiaceae) & 9,00 & 0,58 & 28,00 & 1,48 & 0,06 & 0,42 & 2,48 \\
\hline Cardiopetalum calophyllum (Annonaceae) & 3,00 & 0,19 & 12,00 & 0,63 & 0,20 & 1,24 & 2,06 \\
\hline Guatteria cauliflora (Annonaceae) & 10,00 & 0,64 & 24,00 & 1,27 & 0,03 & 0,24 & 2,05 \\
\hline Parkia sp. (Mimosoideae) & 10,00 & 0,64 & 24,00 & 1,27 & 0,03 & 0,21 & 2,02 \\
\hline Croton sp. (Euphorbiaceae) & 12,00 & 0,77 & 16,00 & 0,85 & 0,07 & 0,44 & 1,98 \\
\hline Tachigali venusta (Caesalpinoideae) & 5,00 & 0,32 & 16,00 & 0,85 & 0,13 & 0,81 & 1,90 \\
\hline Tachigali sp. (Caesalpinoideae) & 3,00 & 0,19 & 8,00 & 0,42 & 0,18 & 1,14 & 1,72 \\
\hline Ocotea glomerata (Lauraceae) & 6,00 & 0,38 & 20,00 & 0,98 & 0,04 & 0,26 & 1,62 \\
\hline Nectrandra sp. (Lauraceae) & 3,00 & 0,19 & 12,00 & 0,63 & 0,13 & 0,83 & 1,60 \\
\hline Abuta concolor (Menispermaceae) & 6,00 & 0,38 & 16,00 & 0,85 & 0,05 & 0,35 & 1,58 \\
\hline Trichilia pallida (Meliaceae) & 8,00 & 0,51 & 16,00 & 0,85 & 0,04 & 0,28 & 1,57 \\
\hline Himatanthus sucuuba (Apocynaceae) & 6,00 & 0,38 & 20,00 & 1,06 & 0,02 & 0,16 & 1,52 \\
\hline Pouroma cecropiifolia (Cecropiaceae) & 3,00 & 0,19 & 12,00 & 0,63 & 0,11 & 0,71 & 1,49 \\
\hline Virola calophylla (Myristicaceae) & 7,00 & 0,45 & 16,00 & 0,85 & 0,03 & 0,19 & 1,41 \\
\hline Alchornea triplinervia (Euphorbiaceae) & 4,00 & 0,26 & 12,00 & 0,63 & 0,07 & 0,48 & 1,37 \\
\hline Protium aracouchini (Burseraceae) & 7,00 & 0,45 & 16,00 & 0,85 & 0,02 & 0,14 & 1,36 \\
\hline Dipteryx odorata (Papilionoideae) & 4,00 & 0,26 & 16,00 & 0,78 & 0,04 & 0,31 & 1,34 \\
\hline Talisia sp. (Sapindaceae) & 6,00 & 0,38 & 16,00 & 0,85 & 0,02 & 0,16 & 1,32 \\
\hline Cordia alliodora (Boraginaceae) & 2,00 & 0,13 & 4,00 & 0,21 & 0,16 & 1,00 & 1,32 \\
\hline Caliptranthes sp. (Myrtaceae) & 4,00 & 0,26 & 12,00 & 0,63 & 0,04 & 0,26 & 1,15 \\
\hline Guapira opposita (Myrtaceae) & 4,00 & 0,26 & 16,00 & 0,85 & 0,01 & 0,08 & 1,11 \\
\hline Vismia brasiliensis (Clusiaceae) & 4,00 & 0,26 & 16,00 & 0,85 & 0,01 & 0,07 & 1,11 \\
\hline Scheflera morototoni (Araliaceae) & 5,00 & 0,32 & 8,00 & 0,42 & 0,05 & 0,36 & 1,06 \\
\hline Sapium glandulosum (Euphorbiaceae) & 3,00 & 0,19 & 8,00 & 0,42 & 0,07 & 0,47 & 1,05 \\
\hline Hevea brasiliensis (Euphorbiaceae) & 5,00 & 0,32 & 12,00 & 0,63 & 0,01 & 0,12 & 1,02 \\
\hline Ecclinusa ramiflora (Sapotaceae) & 3,00 & 0,19 & 12,00 & 0,63 & 0,03 & 0,23 & 1,01 \\
\hline Goupia glabra (Celastraceae) & 7,00 & 0,45 & 8,00 & 0,42 & 0,02 & 0,15 & 0,99 \\
\hline Astronium sp. (Anacardiaceae) & 3,00 & 0,19 & 12,00 & 0,63 & 0,01 & 0,11 & 0,93 \\
\hline Protium heptaphyllum (Burseraceae) & 4,00 & 0,26 & 12,00 & 0,63 & 0,01 & 0,09 & 0,92 \\
\hline Sorocea guilleminiana (Moraceae) & 3,00 & 0,19 & 12,00 & 0,63 & 0,02 & 0,14 & 0,92 \\
\hline Inga dysantha (Mimosoideae) & 3,00 & 0,19 & 12,00 & 0,63 & 0,01 & 0,08 & 0,85 \\
\hline Pseudolmedia laevigata (Moraceae) & 2,00 & 0,13 & 8,00 & 0,42 & 0,05 & 0,34 & 0,85 \\
\hline Neea oppositifolia (Nyctaginaceae) & 4,00 & 0,26 & 8,00 & 0,42 & 0,03 & 0,19 & 0,84 \\
\hline Siparuna guianensis (Monimiaceae) & 3,00 & 0,19 & 12,00 & 0,63 & 0,00 & 0,06 & 0,83 \\
\hline Protium sp. (Burseraceae) & 2,00 & 0,13 & 8,00 & 0,42 & 0,04 & 0,29 & 0,80 \\
\hline Palicourea guianenis (Rubiaceae) & 4,00 & 0,26 & 8,00 & 0,42 & 0,01 & 0,09 & 0,73 \\
\hline Fagara rhoifolia (Rutaceae) & 2,00 & 0,13 & 4,00 & 0,20 & 0,05 & 0,37 & 0,69 \\
\hline Hyeronima alchorneoides (Euphorbiaceae) & 1,00 & 0,06 & 4,00 & 0,00 & 0,06 & 0,38 & 0,64 \\
\hline Duguetia lanceolata (Annonaceae) & 3,00 & 0,19 & 8,00 & 0,42 & 0,00 & 0,06 & 0,64 \\
\hline Aniba ferrea (Lauraceae) & 2,00 & 0,13 & 8,00 & 0,42 & 0,01 & 0,08 & 0,63 \\
\hline Tetragastris sp. (Burseraceae) & 3,00 & 0,19 & 8,00 & 0,39 & 0,00 & 0,03 & 0,61 \\
\hline Miconia sp. (Melastomataceae) & 3,00 & 0,19 & 8,00 & 0,42 & 0,00 & 0,03 & 0,61 \\
\hline Eugênia sp. (Myrtaceae) & 2,00 & 0,13 & 8,00 & 0,42 & 0,01 & 0,07 & 0,59 \\
\hline Guatteria nigrescens (Annonaceae) & 2,00 & 0,13 & 8,00 & 0,42 & 0,00 & 0,04 & 0,55 \\
\hline Duguettia sp. (Annonaceae) & 2,00 & 0,13 & 8,00 & 0,42 & 0,00 & 0,04 & 0,55 \\
\hline Guatteria sp. (Annonaceae) & 2,00 & 0,13 & 8,00 & 0,42 & 0,00 & 0,03 & 0,55 \\
\hline Palicourea sp. (Rubiaceae) & 2,00 & 0,13 & 8,00 & 0,42 & 0,00 & 0,03 & 0,54 \\
\hline Cupania sp. (Sapindaceae) & 2,00 & 0,13 & 8,00 & 0,42 & 0,00 & 0,02 & 0,54 \\
\hline Caryocar brasiliense (Caryocaraceae) & 3,00 & 0,19 & 4,00 & 0,21 & 0,01 & 0,10 & 0,48 \\
\hline Aparisthmium cordatum (Euphorbiaceae) & 1,00 & 0,06 & 0,04 & 0,00 & 0,06 & 0,39 & 0,45 \\
\hline Protium pilosum (Burseraceae) & 2,00 & 0,13 & 0,08 & 0,00 & 0,00 & 0,29 & 0,42 \\
\hline
\end{tabular}


Tabela 2 - Continuação

\begin{tabular}{|c|c|c|c|c|c|c|c|}
\hline Nome Científico & $\mathrm{DA}$ & DR & FA & FR & DOA & DOR & $\mathrm{Vl}$ \\
\hline Triplaris sp. (Sapindaceae) & 2,00 & 0,13 & 0,04 & 0,00 & 0,00 & 0,29 & 0,42 \\
\hline Reedhia sp. (Clusiaceae) & 2,00 & 0,13 & 0,04 & 0,02 & 0,00 & 0,29 & 0,42 \\
\hline Eriotheca gracilipes (Bombacaceae) & 2,00 & 0,13 & 4,00 & 0,21 & 0,01 & 0,08 & 0,39 \\
\hline Quiina pteridophylla (Quiinaceae) & 1,00 & 0,06 & 0,04 & 0,00 & 0,02 & 0,14 & 0,39 \\
\hline Micropholis sp. (Sapotaceae) & 1,00 & 0,06 & 0,04 & 0,00 & 0,01 & 0,10 & 0,36 \\
\hline Ecclinusa sp. (Sapotaceae) & 1,00 & 0,06 & 0,04 & 0,00 & 0,01 & 0,10 & 0,35 \\
\hline Cheiloclinium cognatum (Hippocrateaceae) & 1,00 & 0,06 & 0,04 & 0,00 & 0,01 & 0,09 & 0,35 \\
\hline Pourouma velutina (Cecropiaceae) & 1,00 & 0,06 & 0,00 & 0,00 & 0,01 & 0,06 & 0,32 \\
\hline Mezilaurus itauba (Lauraceae) & 1,00 & 0,06 & 0,00 & 0,00 & 0,00 & 0,05 & 0,31 \\
\hline Bowdichia virgilioides (Papilionoideae) & 1,00 & 0,06 & 4,00 & 0,00 & 0,03 & 0,24 & 0,30 \\
\hline Pera glabrata (Euphorbiaceae) & 1,00 & 0,06 & 0,04 & 0,00 & 0,00 & 0,03 & 0,29 \\
\hline Trema micrantha (Ulmaceae) & 1,00 & 0,06 & 0,04 & 0,00 & 0,00 & 0,04 & 0,29 \\
\hline Psychotria capitata (Rubiaceae) & 1,00 & 0,06 & 0,04 & 0,00 & 0,00 & 0,03 & 0,28 \\
\hline Cupania castaneifolia (Sapindaceae) & 1,00 & 0,06 & 0,04 & 0,00 & 0,00 & 0,03 & 0,28 \\
\hline Inga vera (Mimosoideae) & 1,00 & 0,06 & 0,00 & 0,00 & 0,00 & 0,03 & 0,28 \\
\hline Duroia saccifera (Rubiaceae) & 1,00 & 0,06 & 0,04 & 0,00 & 0,00 & 0,01 & 0,27 \\
\hline Cordia nodosa (Boraginaceae) & 1,00 & 0,06 & 0,04 & 0,00 & 0,00 & 0,01 & 0,27 \\
\hline Xylopia emarginata (Annonaceae) & 1,00 & 0,06 & 0,04 & 0,00 & 0,00 & 0,01 & 0,27 \\
\hline Lacistema pubescens (Lacistemataceae) & 1,00 & 0,06 & 0,04 & 0,00 & 0,00 & 0,01 & 0,27 \\
\hline Toulicia sp. (Sapindaceae) & 1,00 & 0,06 & 0,04 & 0,00 & 0,00 & 0,01 & 0,27 \\
\hline Calophyllum brasiliense (Clusiaceae) & 1,00 & 0,06 & 0,04 & 0,02 & 0,02 & 0,13 & 0,19 \\
\hline Bombacopsis glabra & 1,00 & 0,06 & 0,04 & 0,00 & 0,01 & 0,11 & 0,17 \\
\hline Alophyllus edulis (Sapindaceae) & 1,00 & 0,13 & 0,00 & 0,00 & 0,00 & 0,03 & 0,16 \\
\hline Persea cordata & 2,00 & 0,13 & 0,04 & 0,00 & 0,00 & 0,03 & 0,16 \\
\hline $\mathrm{NI}$ & 2,00 & 0,13 & 0,08 & 0,00 & 0,01 & 0,02 & 0,15 \\
\hline Byrsonima sp. (Malpighiaceae) & 1,00 & 0,06 & 0,04 & 0,00 & 0,00 & 0,05 & 0,11 \\
\hline Calyptranthes paniculata & 1,00 & 0,07 & 0,08 & 0,00 & 0,00 & 0,03 & 0,10 \\
\hline Abuta grandifolia & 1,00 & 0,06 & 0,04 & 0,00 & 0,00 & 0,03 & 0,09 \\
\hline Bocageopsis multiflora & 1,00 & 0,06 & 0,04 & 0,00 & 0,00 & 0,03 & 0,09 \\
\hline Dalbergia sp. & 1,00 & 0,06 & 0,04 & 0,00 & 0,00 & 0,03 & 0,09 \\
\hline Couepia sp. & 1,00 & 0,06 & 0,04 & 0,00 & 0,00 & 0,03 & 0,09 \\
\hline Abarema jupunba (Mimosoideae) & 1,00 & 0,06 & 0,04 & 0,00 & 0,00 & 0,02 & 0,08 \\
\hline Apeiba echinata (Tiliaceae) & 1,00 & 0,06 & 0,04 & 0,00 & 0,00 & 0,02 & 0,08 \\
\hline TOTAL & 1.555 & 99,74 & $1.889,44$ & 98,62 & 15,55 & 100,46 & 295,54 \\
\hline
\end{tabular}

Dentre as espécies existem diversas generalistas, que ocorrem em florestas vinculadas à umidade como Cheiloclinium cognatum, Callophyllum brasiliense, Protium heptaphyllum, outras que são exclusivas de formaçôes savânicas do cerrado como Caryocar brasiliense e outras tipicamente amazônicas como Mezilaurus itauba evidenciando uma flora mista nesta área de transição, também em nível específico.

\section{CONCLUSÕES}

A composição florística e estrutura do fragmento, confirma a classificação da área como floresta de transição efetuada com base no mapa de vegetação do Brasil, escala 1: 5 milhóes, com a presença de espécies arbóreas generalistas, que se adaptam tanto em Floresta Amazônica quanto em Cerrado e de espécies exclusivas de Cerrado e de Floresta Amazônica.

Espécies do gênero Cecropia apresentaram uma alta densidade e freqüência e o maior índice Valor de Importância neste trabalho (Cecropia sp.), sugerindo que a área estudada está em processo de sucessão secundária. A presença desse grupo botânico indica que a área está em processo de recuperação. Cecropia é um gênero importante na recomposição de florestas ciliares, recuperação de áreas degradadas, sombreamento para estabelecimento de espécies arbóreas secundárias tardias ou clímax (Gandolfi et al., 1995; Felfili et al., 2000) podendo ser considerado pelo seu pioneirismo como um indicador de perturbaçôes antrópicas. 
Esta floresta apresenta espécies com grande potencial para uso múltiplo, como as madeireiras Mezilaurus itauba (itaúba), Nectandra sp. (louro), Ocotea sp. (canela), Dipteryx odorata (champanhe) e Aspidosperma sp. (peroba) e as frutíferas com grande apreciação popular como pequi e pitomba (Tabela 1). Como reserva urbana pode oferecer benefícios indiretos e ser um espaço demonstrativo para educação ambiental além de proteger essas espécies.

\section{AGRADECIMENTOS}

Ao programa de Pós-graduação em Ciências Florestais e Ambientais da Universidade Federal de Mato Grosso. Ao $\mathrm{CNPq}$ pela bolsa de produtividade em pesquisa à terceira autora e pelo apoio ao Centro de Referência em Conservaçáo da Natureza e Recuperação de Áreas Degradadas da Universidade de Brasília e ao INCT de madeiras da amazônia onde seus projetos estão incluídos. A Christopher William Fagg pela revisão do texto em inglês.

\section{BIBLIOGRAFIA CITADA}

Alencar, A.; Nepstad, N.; Mcgrath, D.; Moutinho, P.; Pacheco, P.; Diaz, M. D. C. V. \& Filho, B. S. 2004. desmatamento na Amazônia: indo além da "emergência crônica". Belém, PA. IPAM

Almeida, S. S.; Lisboa, P. L. B. \& Silva, A. S. 1993. Diversidade florística de uma comunidade arbórea na estação científica "Ferreira Pena", em Caxiuanã (Pará). Bolm. Mus. Paraense Emilio Goeldi, Ser. Botanica, 9: 93-128.

Amaral, I. L; Matos, F. D. A. \& Lima, J. 2000. Composição florística e estrutural de um hectare de floresta densa de terra firme no Rio Uatumã, Amazônia, Brasil. Acta Amazonica, 30: 377-392.

Andrade, L. A. Z.; Felfili, J. M. 2002. Fitossociologia de um fragmento de cerrado sensu stricto na APA do Paranoá, DF, Brasil. Acta Botanica Brasilica, 16: 225-240.

Brasil. Departamento Nacional da Produçáo Mineral. Projeto RADAM. Folha SC21. Juruena. Rio de Janeiro, 1979.

Brower, J. E. \& Zar, J. H. Field e laboratory methods for general ecology. 2 ed. Munique: Win. C. Brown Publishers, 1977. 226p.

Cardoso-Leite, E.; Monteiro, R.; Pagani, M. I. \& Hamburger, D. S. 2002. Fitosifionomia, fitossociologia e conservação da vegetação na Reserva Biológica da Serra do Japi, Jundiaí, SP. Revista Naturalia, 27: 165-200.

Carmo, J. B.; Keller, M. M.; Dias, J. D.; Camargo, P. B. de \& Crill, P. M. 2006. A source of methane from upland forest in the Brazilian Amazon. Geophysical Research Letters, v. 33.

Cientec. Software Mata Nativa 2: Sistema para Análise Fitossociológica, Elaboração de Inventários e Planos de Manejo de Florestas Nativas. Viçosa - MG: cientec, 2006. Disponível em: www. matanativa.com.br. Acesso em 23 de agosto de 2007.

Costa, R. B; Scariot, A. 2003. A fragmentação florestal e os recursos genéticos. In:Fragmentação florestal e alternativas de desenvolvimento rural na Região Centro-Oeste. Costa, R. B. (Org.), p.53. UCDB. Campo Grande.

Cronquist, A. An integrated system of classification of flowering plants. Columbia Univ. Press, New York.1981.

Damasceno Junior, G. A.; Semir, J. Santos, F. A. M. \& Leitão-Filho, H. F. 2005. Structure, distribuition of species and inundation in riparian Forest of Rio Paraguai, Pantanal, Brazil. Flora, 200:119-135.

Felfili, J.M. 1997. Comparison of the dynamics of two gallery forests in Central Brasil. In: Proceedings: International symposium on assessment and monitoring of forests in tropical dry regions with special reference to gallery forests. Imanã-Encinas. J. \& Kleinn C. (Orgs.). p.115-124. University of Brasilia. Brasília.

Felfili, J.M.; Ribeiro, J.F.; Fagg, C.W.; Machado, J.W.B. 2000. Recuperação de Matas de Galeria. Série Documentos, Embrapa Cerrados. Planantina. n.21. 45 p.

Felfili, J. M.; Rezende, A. V.; Silva Junior, M.C. \& Silva, M. A. 2000. Changes in the floristic composition of cerrado senso stricto in Brazil over a nine-year period. Journal of Tropical Ecology, 16: 579-590.

Felfili, M. C. \& Felfili, J. M. 2001. Diversidade alfa e beta no cerrado sensu strict da Chapada Pratinha, Brasil. Acta Botanica Brasílica, 15: $243-270$

Felfili, J. M.; Silva Júnior, M. C.; Sevilha, A. C.; Fagg, C. W.; Walter, B. M. T.; Nogueira, P. E. \& Rezende, A. V. 2004. Diversity, floristic and structural patterns of cerrado vegetation in Central Brazil. Plant Ecology, 175: 37 - 46.

Ferreira Júnior, E. V.; Soares, T. S.; Costa, M.F.F. \& Silva, V.S.M. 2008. Composição, diversidade e similaridade florística de uma floresta tropical semidecídua submontana em Marcelândia - MT. Acta Amazônica 38(4) 2008: 673 - 680.

Gandolfi, S.; leitão Filho, H.F.; Bezerra, C.L.Levantamento florístico e caráter sucessional das espécies arbustivo-arbóreas de uma floresta mesófila semidecídua no município de Guarulhos, SP. Rev. Brasil. Biol., 55:753-767.

Haidar, R. F; Felfili, J. M.; Fagg, C. W. \& Pinto, J. R. R. 2005. Fitossociologia da vegetação arbórea em fragmentos de floresta estacional, Np Parque Ecológico Altamiro de Moura Pacheco, GO. Boletim do Herbário Ezchias Paulo Heringer, 15: 19-46.

Higuchi, N.; Chambers, J. Q.; Santos, J.; Pinto, A. C. M.; Silva, R. P.; Rocha, R. M. \& Tribuzy, E. S. 2004. Dinâmica do carbono da vegetação primária da Amazônia Central. Floresta, 34: 295304 .

Ivanauskas, N. M. \& Rodrigues, R. R. 2000. Florística e fitossociologia de remanescentes de floresta estacional decidual em Piracicaba, São Paulo, Brasil. Revista Brasil. de Botânica, 23:291-304.

Ivanauskas, N.M.; Monteiro, R,; Rodrigues, R.R. 2004. Estrutura de um trecho de floresta amazônica na Bacia do Alto Rio Xingu. Acta Amazônica, 34 (2): 275-299.

Kent, M. \& Coker, P. 1992. Vegetation description analyses. Behaven Press. London. 363p.

Maia, G.N. 2004. Caatinga: árvores e arbustos e suas utilidades. São Paulo. D\&Z Computação Gráfica e Editora. 413 p. 
Kunz, S.H.; Ivanauskas, N. M.; Martins, S. V.; Silva, E. \& Stefanello, D. 2008. Aspectos florísticos e fitossociológicos de um trecho de Floresta Estacional Perenifolia na Fazenda Trairão, Bacia do rio das Pacas, Querência-MT. Acta Amazônica 38:245-254.

Marimon, B. S.. M.; Lima, E. S. 2002. Floristics and phytosociology of the gallery Forest of the Bacaba stream, nova Xavantina, mato grosso, Brazil. Edinburgh Journal of Botany, 59 (2): 303-318.

Marimon, B. S.; Felfili, J. M.; Haridasan, M. 2001․ Studies in monodominant forests in eastern Mato Grosso Brazil: I. A forest of Brosimum rubescens Taub. Edinburgh Journal of Botany, 58(1):123-137.

Marimon, B.S., Felfili, J. M.; Haridasan, M. 2001. Studies in monodominant forests in Eastern Mato grosso, Brazil: II. A forest in the Areóes Xavante Reserve. Edinburgh Journal of Botany, 58 (3): 483-497.

Marimon, B.S.; Lima, E.S.; Duarte, T.G.; Chieregatto, L.C.\& Ratter, J.A. 2006. Observations on the vegetation of Northeastern Mato Gross,Brazil. IV. An Analysis of the Cerrado-Amazonian forest ecotone. Edinburg Journal of Botany, 63: 323-341.

Mata, M. F.; Felix, L. P. 2007. Flora da Paraíba, Brasil: Inga Mill. (Leguminosae-mimosoideae). Revista Brasil. de Biociências, 5(2): 135-137.

Meira Neto, J. A. A; Martins, F. R. 2000. Estrutura da mata da Silvicultura, uma floresta estacional semidecidual Montana no município de Viçosa, MG. Revista Árvore, 21(2): 151-160.

Mobot, Missouri Botanical Garden. W Tropicos. Disponível em http://mobot.mobot. Org/W3T/search/vast.html. Acesso: 2008.

Mueller-Dombois, D.; Elemberg, H. 1974. Aims and methods of vegetation ecology. Willey and Sons. New York.

Muniz, F. H.; Cesar, O. \& Monteiro, R. 1994. Fitossociologia da vegetação arbórea da Reserva Florestal do Sacavém, São Luís, Maranhão (Brasil). Acta Amazonica, 24: 219-236.

Nunes, S. R. D. F. S., Garcia, F. C. P., Lima, H. C. \& CarvalhoOkano, R. M. 2007. Mimosoideae (Leguminosae) arbóreas do Parque Estadual do Rio Doce, Minas Gerais, Brasil: Distribuição geográfica e similaridade florística na Floresta Atlântica no sudeste do Brasil. Rodriguésia, 58: 403-421.

Odum, E. P. :1988. Ecologia. Trad. Ricardo Iglesias Rios e Christopher J. Tribe. Rio de Janeiro.
Oliveira, A. N.; Amaral, I. L. 2004. Florística e fitossociologia de uma floresta de vertentes na Amazônia Central, Amazonas, Brasil. Acta Botânica, 34(1):21-34.

Oliveira, E. C. L.; Felfili, J. M. 2005. Estrutura e dinâmica da regeneração natural de uma mata de galeria no Distrito Federal, Brasil. Acta bot. bras. 19(4): 801-811.

Pereira-Silva, E. F. L.; Santos, J. E.; Kageyama, P. Y. \& Hardt, E. 2004. Florística e fitossociologia dos estratos arbustivos e um arbóreo de um remanescente de cerradão em uma Unidade de Conservação do Estado de São Paulo. Revista Brasil. de botânica, 27: 533-544.

Primack, R. B; Rodrigues, E. 2001. Biologia da conservação. Londrina.

Redling, J. S. H.; Moreno, M. R.; Bonafiman, G. S. L.; Oliveira, N. E. M. \& Gonçalves, M. A. M. 2002. Fitossociologia da Formação Halófila e Psamófila-reptante da Restinga de Praia das Neves, Presidente Kennedy, ES. Cadernos Camilliani, 3: 19-24.

Silva, A. S. L.; Lisboa, P. L. B.; Maciel, U. N. 1992. Diversidade florística e estrutura em floresta densa da bacia do Rio JuruáAM. Boletim: Museu Paraense Emílio Goeldi: Botânica, 8(2):203258.

Silva, C.T.; Reis, G.G.; Reis, M.G.F.; Chaves, R.A. 2004. Avaliação temporal da florística arbórea de uma floresta secundária no município de Viçosa, Minas Gerais. Revista Árvore, 28: 429441.

Silva, N.R.S.; Martins, S. V. \& Meira Net, J. A A. 2004. A Composição florística e estrutura de uma floresta estacional semidecidual Montana em Viçosa, MG. Revista Árvore, 28:397405.

Stein, V.; Paiva, R., Nogueira, R. C., Justo, C. F.; Nery, F. C. \& Nicioli, P. M. 2007. Viabilidade do grão de pólen e efeito do PVP na calgênese de Inga vera Willd. Subsp. affinis (DC.) T. D. Penn. Revista Brasil. de Biociências, 5: 702-704.

Teixeira, L.; Rosendo, J.S. 2004. A expansão da fronteira agrícola no norte do Mato Grosso: impactos sócio-ambientais da exploraçáo madeireira. Sociedade \& Natureza, 16: 71-79.

Recebido em 15/02/2009

Aceito em 15/06/2009 
